\title{
Classical Mathematical Integrated Information Theory
}

\begin{abstract}
This paper is an attempt to give mathematical structure to classical integrated information theory (IIT) by Masafumi Oizumi, Larissa Albantakis, Giulio Tononi, using the definition provided by Giulio Tononi and making few assumptions like the mechanisms are open subset of set of elements and system is the topology of the set of elements. This would make IIT accessible to a wide audience, with more formal basis who can then apply it to non-biological objects too.
\end{abstract}

\section{Introduction}

In the following sections we will develop a mathematical theory of consciousness of a system, by considering definitions from Integrated Information Theory [1]. And there by using classical definition and generating their mathematical enalog.

In the following sections we will review the IIT 3.0 and at the same time try to give what the components of IIT 3.0 is synonymous to in mathematics and thereby giving a mathematical structure to the theory. The statements in the section below in italic is from IIT 3.0, [1] and in regular font is the mathematical construct.

\section{Postulates}

- "EXISTENCE: Mechanisms in a state exist. A system is a set of mechanisms."

It is not difficult to see that if we consider a set of elements $\Lambda$ then system is the topology (the set of open sets), $\mathcal{T}$ of the set $\Lambda$. Mechanisms, $M$ is open subset of set $\Lambda$ i.e. $M$ belongs $\mathcal{T} ; M \in \mathcal{T}$. The state is the way in which elements of a mechanism are arranged. Where elements could be the object exchanging information, for example, neurons. The topology taken by Tononi is discrete topology but any other topology can also be taken.

- "COMPOSITION: Elementary mechanisms can be combined into higher order ones.

The above mathematical structure satisfies the second postulate also. Since mechanisms are open set and union of open sets is a open set, hence is a mechanism.

\section{Structure of Mechanisms}

- "INFORMATION: ....., a mechanism in a state generates information only if it constrains the states of a system that can be its possible causes and effects - its causeeffect repertoire. The more selective the possible causes and effects, the higher the cause-effect information (cei) specified by the mechanism" 
- Definition: Cause-effect repertoire: The probability distribution of potential past and future states of a system as constrained by a mechanism in its current state.

It is self-explanatory, cause-effect repertoire is set of all the probability distributions of states of mechanisms called Perviews, $P$ knowing that mechanism, $M$ is in state, $s$ mathematically speaking $\left\{p^{x}\left(P \mid M_{S}\right)\right\}_{\substack{P \in \mathcal{T} \\ P \neq M}}$ where $P \in \mathcal{T}$ and $P \neq M, p$ represents probability distribution function, $x \in\{e, c\}$ where $e$ represents effect and $c$ represents cause.

- Definition: Cause information (ci) and effect information (ei): Information about the past and the future, which is measured as the distance between the cause repertoire and the unconstrained cause repertoire (same on the effect side).

Let $\mathcal{D}$ be a distance function over probability distribution space. Then the information is defined as follows

$$
x i\left(P \mid M_{s}\right):=\mathcal{D}\left(p^{x}\left(P \mid M_{S}\right), p^{x}(P \mid \varnothing)\right)
$$

Where $p^{x}\left(P \mid M_{S}\right)$ is cause repertoire (effect repertoire), unconstrained cause repertoire (effect repertoire), $p^{x}(P \mid \emptyset)$ and $x \in\{e, c\}$.

- Definition: Cause-effect information (cei): The amount of information specified by a mechanism in a state, measured as the minimum of cause information (ci) and effect information (ei).

Cause-effect information of a mechanism is minimum of cause information and effect information, i.e. for a mechanism $M$ in state, $s$, the cause-effect information, with respect to a perview, $P$ is given by:

$$
\operatorname{cei}\left(P \mid M_{S}\right):=\min \left\{e i\left(P \mid M_{S}\right), \operatorname{ci}\left(P \mid M_{S}\right)\right\}
$$

- "INTEGRATION: A mechanism can contribute to consciousness only if it specifies a cause-effect repertoire (information) that is irreducible to independent components. Integration/irreducibility $\varphi$ is assessed by partitioning the mechanism and measuring what difference this makes to its cause-effect repertoire."

Let $\mathcal{p}^{x}\left(P \mid \widehat{M}_{s}, \breve{M}_{s}\right)$ be probability distribution of states of states of system $\mathcal{T}$ knowing that mechanism $\widehat{M}$ and $\breve{M}$ are in state $s$. Where $\widehat{M} \cup \breve{M}:=M, \widehat{M}, \breve{M} \neq M$ and $\widehat{M}, \breve{M} \in \mathcal{T}$ then,

$$
\varphi^{x}\left(P \mid M_{s}\right):=\min \left\{\mathcal{D}\left(p^{x}\left(P \mid M_{s}\right), p^{x}\left(P \mid \widehat{M}_{s}, \widetilde{M}_{s}\right)\right)\right\}_{\widehat{M} \cup \breve{M}:=M}
$$

Where $\varphi^{x}$ is irreducibility and we can justify this mathematical expression on the basis that it is the minimum distance of repertoire of a mechanism $M$, in state $s$, i.e. $p^{x}\left(P \mid M_{S}\right)$ 
from the repertoire of its constituents, i.e. $p^{x}\left(P \mid \widehat{M}_{s}, \breve{M}_{s}\right)$ which is synonymous to the definition of irreducibility. Where $x \in\{e, c\}$ and integrated cause-effect information of mechanism, $M$ and in state, $s$ is;

$$
\varphi\left(P \mid M_{S}\right):=\min \left\{\varphi^{e}\left(M_{S}\right), \varphi^{c}\left(M_{S}\right)\right\}
$$

- "EXCLUSION: A mechanism can contribute to consciousness at most one cause-effect repertoire, the one having the maximum value of integration/irreducibility $\varphi^{\max }(s)$. This is its maximally irreducible cause-effect repertoire (MICE, or quale sensu stricto.) If the MICE exist, the mechanism constitutes a concept."

It is plain and simple to see MICE,

$$
\varphi^{\max }\left(M_{S}\right):=\max \left\{\varphi\left(P \mid M_{S}\right)\right\}_{\substack{P \in \mathcal{T} \\ P \neq M}}
$$

That is maximum of all the integrated cause-effect information taken over all the preview not equals to mechanism, $M$. If $\varphi^{\max }\left(M_{S}\right)>0$ then it constitutes a concept, which is defined as follows

$$
\mathcal{C}\left(M_{S}\right):=\left(\varphi^{\max }\left(M_{S}\right), p^{c}\left(M_{S}\right), p^{e}\left(M_{S}\right)\right)
$$

\section{Systems of mechanisms}

- INFORMATION:

A conceptual structure is a constellation of points in concept space, where each axis is a possible past/future state of the set of elements, and each point is a concept ...... The higher the number of different concepts and their $\varphi^{\text {max }}$ value, the higher the conceptual information $\mathcal{C J}$ that specifies a particular constellation and distinguishes it from other possible constellations

Conceptual structure as explained above is a collection of tuples of concepts over all the future and past states and the collection is over the elements of subsystem, that is Conceptual structure, $\mathcal{C} \mathcal{S}(\mathcal{U})$ for a subsystem, $\mathcal{U} \subset \mathcal{T}$, is defined as:

$$
\mathcal{C} \mathcal{S}(\mathcal{U}):=\left\{\left(\mathcal{C}\left(M_{s}\right)\right)_{s \in \mathbb{S}}\right\}_{M \in \mathcal{U}}
$$

Where $\mathbb{S}$ is set of all possible state. The conceptual information, $\mathcal{C J}$ of conceptual structure, $\mathcal{C} \mathcal{S}(\mathcal{U})$ is linear functional on space of conceptual structure class and is defined as:

$$
\mathcal{C J}(\mathcal{C S}(\mathcal{U})):=\sum_{\substack{M \in \mathcal{U} \\ s \in \mathbb{S}}} \varphi^{\max }\left(M_{s}\right) \mathcal{J}(\mathcal{U})
$$


Where $\mathcal{J}(\mathcal{U}):=\max \left\{\operatorname{cei}\left(P \mid M_{S}\right)\right\}_{\substack{P \in \mathcal{T} \\ M \in \mathcal{U}}}$. This mathematical expression suites the definition as, it is increases with increase in number of points and also it increases with $\varphi^{\max }$.

- INTEGRATION: A set of elements can be conscious only if its mechanisms specify a conceptual structure that is irreducible to non-interdependent components (strong integration). Strong integration/irreducibility $\Phi$ is assessed by partitioning the set of elements into subsets with unidirectional cuts.

let $\Delta$ be a distance function or metric over class of conceptional structure and could be defined as follows:

$$
\Delta(\mathcal{C S}(\mathcal{U}), \mathcal{C S}(\mathcal{V})):=|\mathcal{C J}(\mathcal{C S}(\mathcal{U}))-\mathcal{C J}(\mathcal{C S}(\mathcal{V}))|
$$

Where $\mathcal{U}, \mathcal{v} \subset \mathcal{T}$

Then strong irreducibility $\Phi(\mathcal{U})$ of a sub-system, $\mathcal{U}$ as defined above is the minimum distance measured by the distance function, $\Delta$ from conceptual structure of sub-system $\mathcal{U}$ and sum of conceptual structure of its constituents, $\mathcal{C} \mathcal{S}(\widehat{\mathcal{U}}), \mathcal{C} \mathcal{S}(\breve{u})$. i.e.

$$
\Phi(\mathcal{U}):=\min \{\Delta(\mathcal{C} \mathcal{S}(\mathcal{U}), \mathcal{C} \mathcal{S}(\widehat{\mathcal{U}})+\mathcal{C} \mathcal{S}(\breve{\mathcal{U}}))\}_{\substack{\hat{u} \cup \breve{u}=u \\ \hat{u}, \breve{u} \neq u \\ \widehat{u}, \breve{u} \subset \mathcal{T}}}
$$

Where minimum is taken over all $\hat{\mathcal{u}}, \breve{u}$, satisfying three conditions:

- $\quad \widehat{u}, \breve{u} \subset T$ : i.e., $\widehat{u}, \breve{u}$ belongs to the system

- $\widehat{u} \cup \breve{u}=\mathcal{U}$ : i.e., union of $\widehat{u}, \breve{u}$ is $\mathcal{U}$

- $\widehat{u}, \breve{u} \neq \mathcal{U}$ : i.e. None of $\widehat{u}, \breve{u}$ equals to $u$

- EXCLUSION: Of all overlapping sets of elements, only one set can be conscious - the one whose mechanisms specify a conceptual structure that is maximally irreducible $(M I C S)$ to independent components. A local maximum of integrated information $\Phi^{\text {max }}$ (over elements, space, and time) is called a complex.

It is synonymous to MICE.

$$
\Phi^{\max }(\mathcal{T}):=\max \{\Phi(\mathcal{U})\}_{\mathcal{U} \subset \mathcal{T}}
$$

The maximum is taken over all the subsets, $\mathcal{U}$ of the system, $\mathcal{T}$. Where $\Phi^{\max }(\mathcal{T})$ is measure of consciousness of system $\mathcal{T}$.

\section{Discussion and Conclusion}

For a set of elements $\Lambda$ (e.g. degrees freedom), its open subset is a mechanism, $M$ (in case of discrete topology all the subsets are open) and set of open sets i.e. topology, $\mathcal{T}$ is system. 
Cause- effect repertoire of a given mechanism, $M$ is the its conditional probably density, $p^{x}\left(P \mid M_{S}\right)$ of other mechanisms called perview, $P$ knowing a given mechanism, $M$ in a given particular state. Given a distance function on probability density space, $\mathcal{D}$. The minimum distance between the repertoire of a mechanism, $p^{x}\left(P \mid M_{S}\right)$ and repertoire of its sub-mechanism and then considering that repertoire as perview for calculating the repertoire for its complement sub-mechanism, $p^{x}\left(P \mid \widehat{M}_{S}, \widetilde{M}_{S}\right)$. The maximum of all cause effect integrated information is called MICE and if it is non zero then it creates concept. The concept is 3-tuple of MICE, respective cause repertoire and effect repertoire. Conceptual structure is a subsystem (subset of topology) is tuple over all states and mechanisms over that system. Conceptual information is measure of information in conceptual structure and increases with increase in MICE of concept of conceptual structure and with increase in number of concepts of conceptual structure and could be defined as:

$$
\mathcal{C J}(\mathcal{C S}(\mathcal{U})):=\sum_{\substack{M \in \mathcal{U} \\ s \in \mathbb{S}}} \varphi^{\max }\left(M_{S}\right) \mathcal{J}(\mathcal{U})
$$

Where $\mathcal{J}(\mathcal{U}):=\max \left\{\operatorname{cei}\left(P \mid M_{S}\right)\right\}_{\substack{P \in \mathcal{T} \\ M \in \mathcal{U}}}$. Integrated conceptual information is the minimum of the distance between conceptual structure of a sub-system, $\mathcal{U}$ and the subtotal of the conceptual structure of its constituents, $\widehat{U}, \breve{u}$ and is synonymous to integrated cause-effect information and can be defined as,

$$
\Phi(\mathcal{U}):=\min \{\Delta(\mathcal{C S}(\mathcal{U}), \mathcal{C} \mathcal{S}(\widehat{\mathcal{u}})+\mathcal{C} \mathcal{S}(\breve{\mathcal{u}}))\}_{\substack{\hat{u} \cup \breve{u}=u \\ \widehat{u}, \breve{u} \neq u \\ \widehat{u}, \breve{u} \subset \mathcal{T}}}
$$

Where $\Delta$ is distance function over space of conceptual structures and can be best defined as,

$$
\Delta(\mathcal{C S}(\mathcal{U}), \mathcal{C S}(\mathcal{V})):=|\mathcal{C J}(\mathcal{C S}(\mathcal{U}))-\mathcal{C J}(\mathcal{C S}(\mathcal{V}))|
$$

Where $\mathcal{U}, \mathcal{v} \in \mathcal{T}$. Maximum of integrated conceptual information is measure of consciousness.

\section{Declarations}

\section{Ethics approval and consent to participate}

Not applicable

\section{Consent for publication}

I give my consent to publication

\section{Availability of supporting data}

Not applicable. If your manuscript does not contain any data, please state 'Not applicable' in this section.

\section{Competing interests}


No competing interests

\section{Funding}

No funding

\section{Authors' contributions}

I solely contributed in every capacity

\section{Acknowledgements}

Not applicable

\section{Reference}

1. Oizumi, M., Albantakis, L., \& Tononi, G. (2014). From the Phenomenology to the Mechanisms of Consciousness: Integrated Information Theory 3.0. PLoS Computational Biology, 10(5). https://doi.org/10.1371/journal.pcbi.1003588

2. Kleiner, J. Mathematical Models of Consciousness. Entropy 2020, 22, 609.

3. Bengtsson, I., \& Zyczkowski, K. (2006). Geometry of Quantum States: An Introduction to Quantum Entanglement. Cambridge: Cambridge University Press. doi:10.1017/CBO9780511535048

4. G. Tononi, BMC Neuroscience 5, 42 (2004).

5. M. Oizumi, L. Albantakis, and G. Tononi, PLOS Computational Biology 10, 5 (2014)

6. K. Kremnizer and A. Ranchin, Found. Phys. 45, 889 (2015).

7. L. Albantakis and G. Tononi, Entropy 17, 5472 (2015).

8. M. Tegmark, Chaos, Solitons \& Fractals 76, 238 (2015).

9. M. Tegmark, PLOS Computational Biology 12, 11 (2016).

10. G. Tononi and C. Koch, Phil. Trans. R. Soc. B 370, 20140167 (2015)

11. E. Lieb, D. Robinson, Commun. Math. Phys. 28, 251 (1972)

12. Sharma, S. (2020, December 18). Entropic Integrated Information Theory-Theory of Consciousness. https://doi.org/10.31219/osf.io/uswmr

13. Munkres, J. R. (2000). Topology. Upper Saddle River, NJ: Prentice Hall, Inc.

14. https://doi.org/10.1007/978-1-4757-1793-8_1

15. Tononi, G. An information integration theory of consciousness. BMC Neurosci 5, 42 (2004). https://doi.org/10.1186/1471-2202-5-42 
16. Science Translational Medicine 14 Aug 2013:Vol. 5, Issue 198, pp. 198ra105 DOI: 10.1126/scitranslmed.3006294 